Supplementary Information

\title{
Facile Preparation of Graphene Oxide Membranes for Gas \\ Separation
}

Chenglong Chi, Xuerui Wang, Yongwu Peng, Yuhong Qian, Zhigang Hu, Jinqiao Dong, and Dan Zhao*

Department of Chemical \& Biomolecular Engineering, National University of Singapore, 4 Engineering Drive 4, 117585 Singapore

Correspondence and requests for materials should be addressed to D.Z. (E-mail: chezhao@nus.edu.sg). 

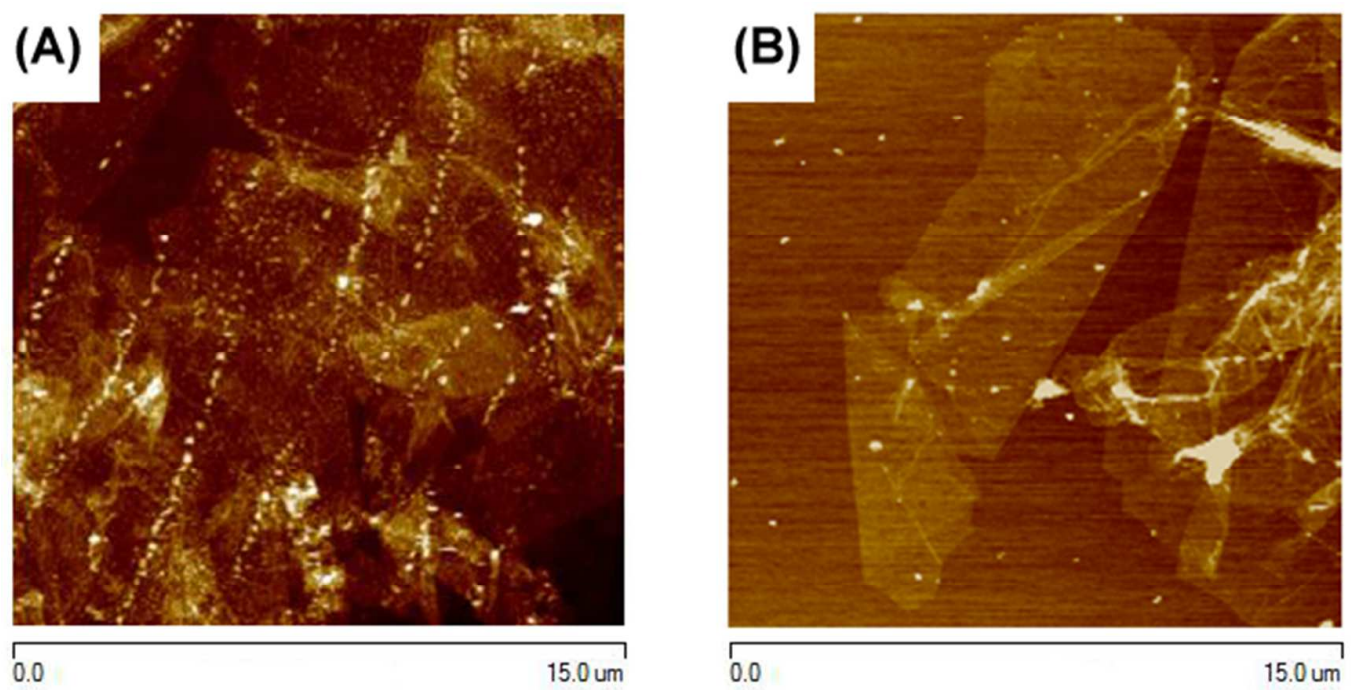

Figure S1. (A) AFM image of exfoliated GO nanosheets before purification; (B) AFM image of exfoliated GO nanosheets after 5 time purification.

Before purification, we can see some smaller GO nanoparticles mixed with larger GO nanosheets. After purification, most of the small GO nanoparticles are removed, leading to increased average size of GO nanosheets seen in Figure 3 and Figure S3. 

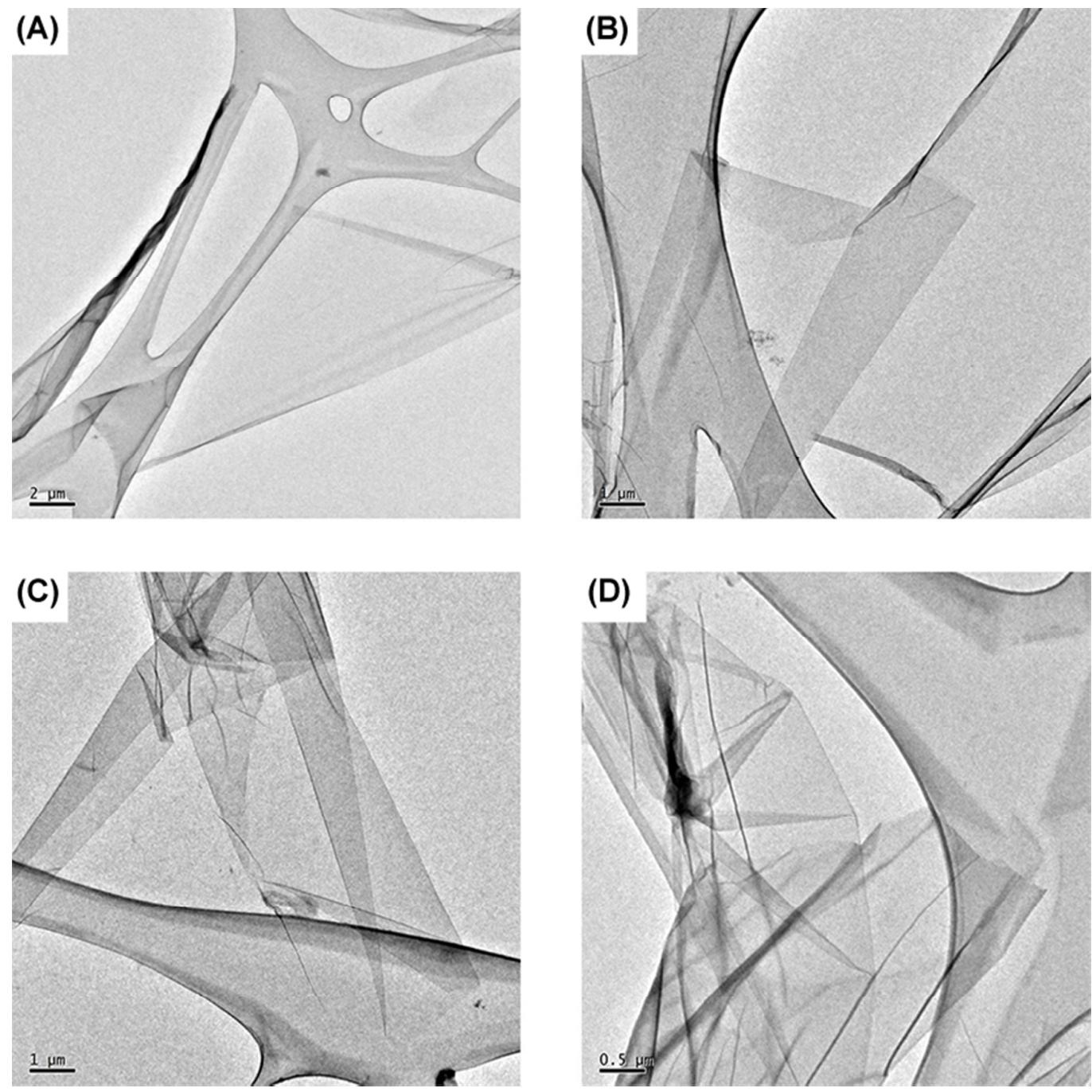

Figure S2. TEM images of exfoliated and purified GO nanosheets.

These TEM images indicate that GO can be successfully exfoliated by the mild freeze-thaw method into few-layered nanosheets with easily curled morphology. 


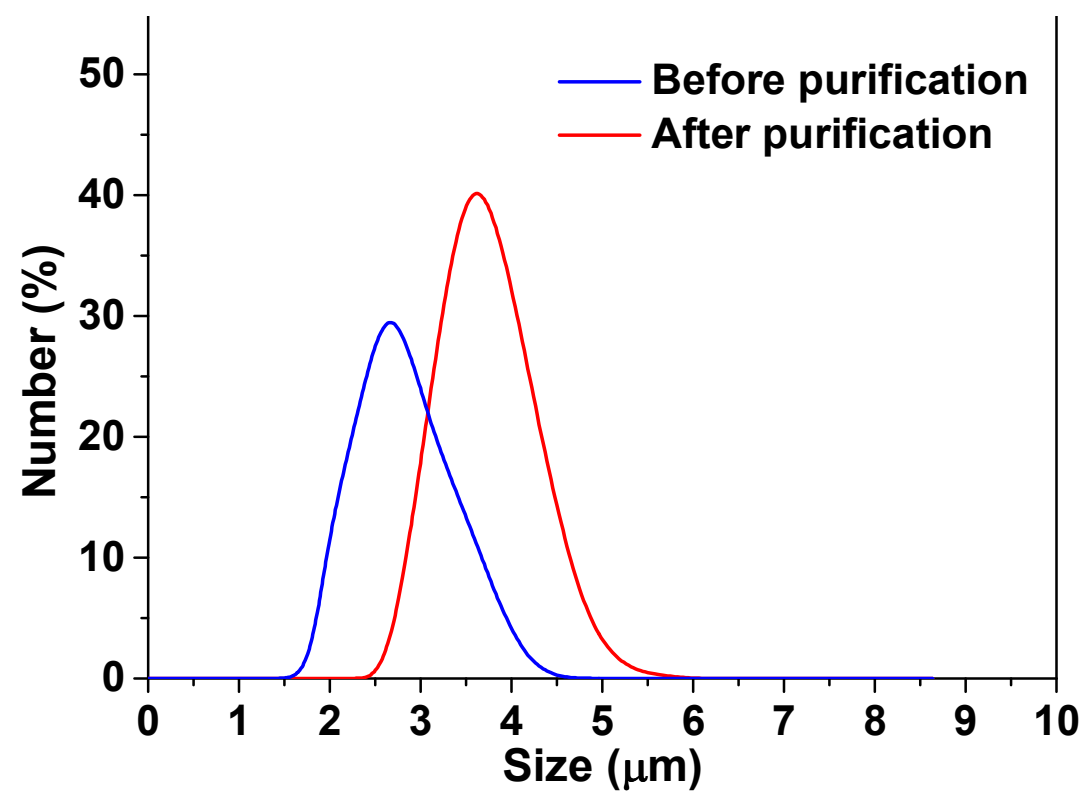

Figure S3. Raw particle size distribution data of GO nanosheets before and after purification.

The average lateral size of GO nanosheets can be increased from $9 \mu \mathrm{m}$ to $13 \mu \mathrm{m}$ after purification because of the removal of small GO nanoparticles. 

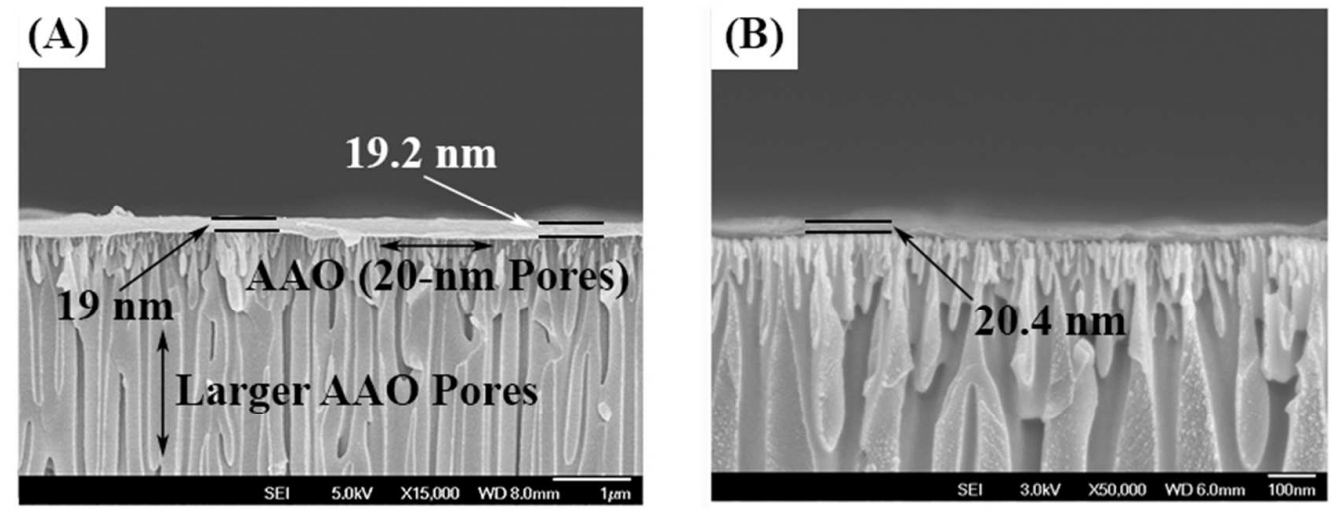

Figure S4. Cross-sectional SEM images of GO membranes prepared by spin coating.

It can be observed from these images that the GO membranes are formed by the stacking of GO nanosheets to a thickness of around $20 \mathrm{~nm}$. 


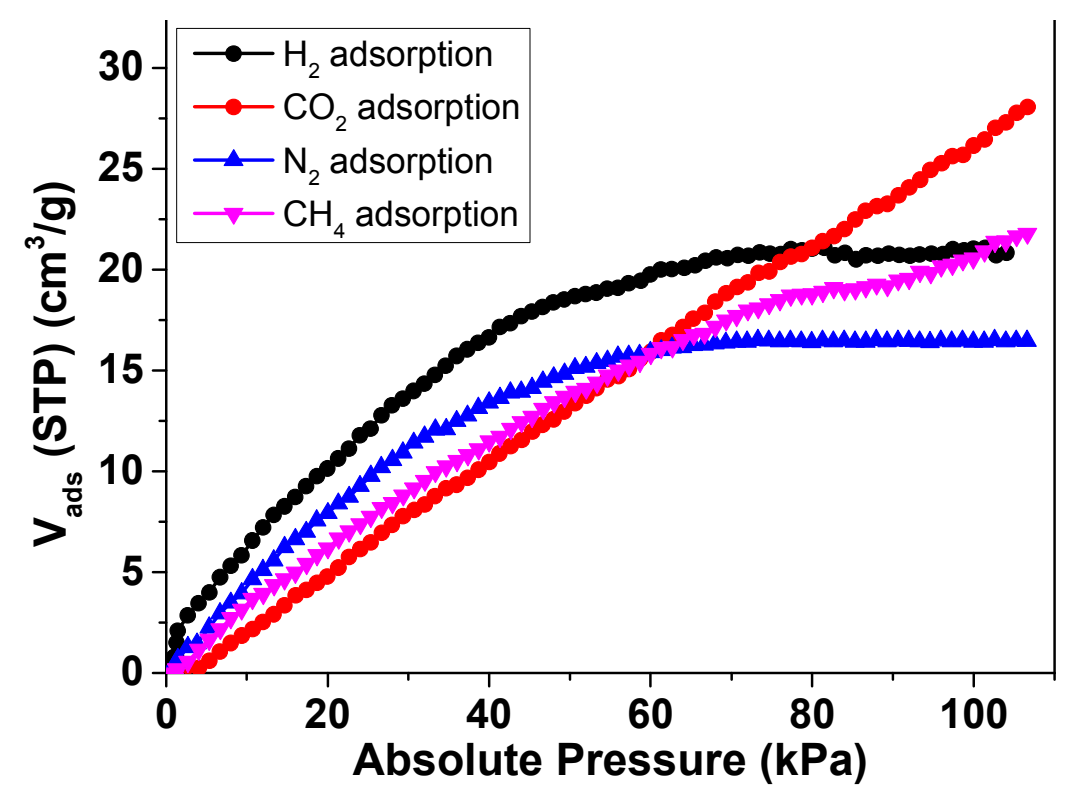

Figure S5. Adsorption isotherms of different gases in GO membrane at $298 \mathrm{~K}$.

This indicates the relatively similar gas sorption amount of various gases into GO membranes under conditions similar to membrane tests $(298 \mathrm{~K}, 1 \mathrm{~atm})$. 


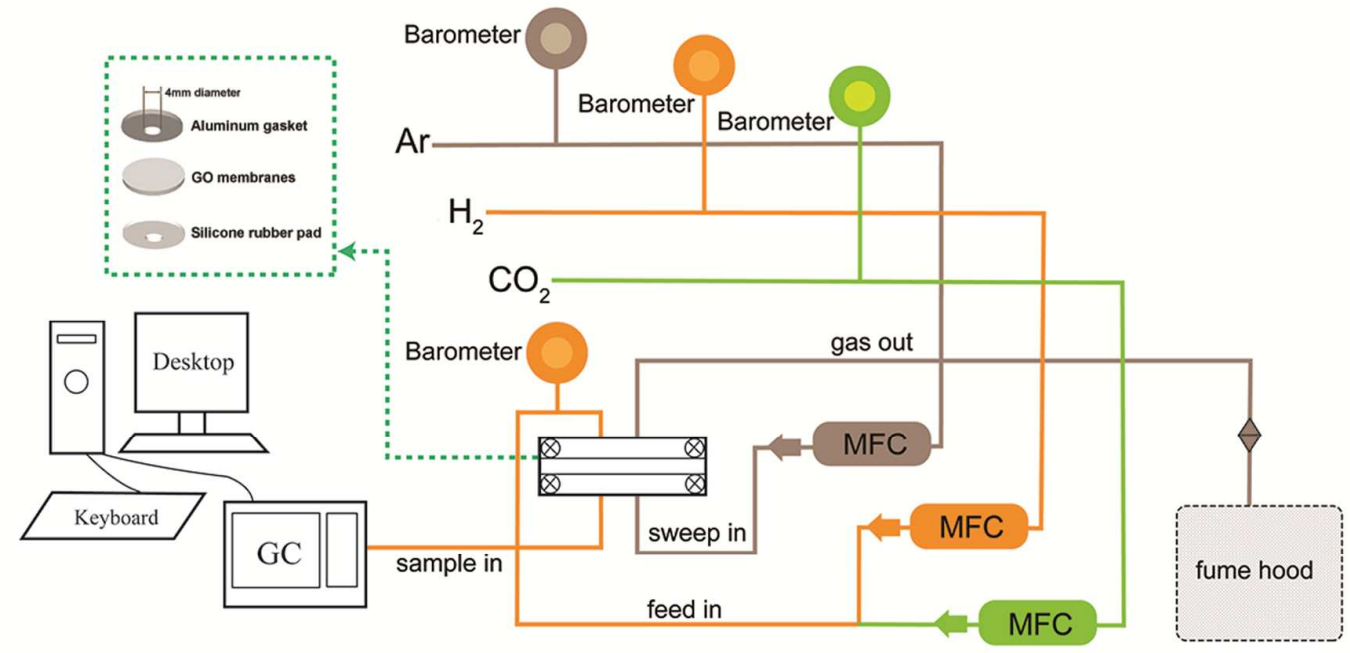

Figure S6. Apparatus scheme of gas separation measurement. 